\title{
BOUNDARY VALUE PROBLEMS FOR FRACTIONAL DIFFERENTIAL EQUATION IN SPECIAL BANACH SPACE
}

\author{
BEDDANI MOUSTAFA $^{1, *}$, HEDIA BENAOUDA ${ }^{2}$ \\ ${ }^{1}$ Department of Mathematics, Djillali Liabes University of Sidi Bel-Abbés, PO BOX 89 22000 Bel-Abbés, \\ Algeria \\ ${ }^{2}$ Laboratory of Mathematics, University of Tiaret, PO BOX 7814000 Tiaret, Algeria
}

${ }^{*}$ Corresponding author: beddani2004@yahoo.fr

\begin{abstract}
This paper studies the existence of solutions of boundary value problem for fractional differential equations on the half-line in a special Banach space. The main result is based on Mönch fixed point theorem combining with a suitable measure of non-compactness, an example is given to illustrate our approach.
\end{abstract}

\section{INTRODUCTION}

Fractional differential equations play a very important role in describing some real world problems, for example, in the description of hereditary properties of various materials and processes. They are also widely applied in the mathematical modeling of processes in physics, chemistry, aerodynamics, electro-dynamics of complex medium, polymer rheology, etc. Consequently, the fractional calculus and its applications in various fields of science and engineering have received much attention and have developed very rapidly (cf. $[18,20,23]$ for instance).

Very recently, many research papers have appeared concerning the fractional differential equations in Banach spaces, some of them investigated the existence results of solutions on finite intervals by classical tools from

Received October $16^{\text {th }}, 2020$; accepted November $11^{\text {th }}, 2020$; published February $24^{\text {th }}, 2021$.

2010 Mathematics Subject Classification. 34B15, 34B40, 26A33.

Key words and phrases. boundary value problem, measure of non-compactness, unbounded domain, special Banach space, Mönch fixed point theorem, Riemann-Liouville fractional derivative.

(C)2021 Authors retain the copyrights of their papers, and all open access articles are distributed under the terms of the Creative Commons Attribution License. 
functional analysis; see, for example References. [1,3, 6-8, 19,21,22].

In [4]. A. Arara and M. Benchohra studied the following problem

$$
\begin{cases}{ }^{c} D_{0^{+}}^{\alpha} y(t)=f(t, y(t)), & t \in J=(0,+\infty), 1<\alpha \leq 2, \\ y(0)=y_{0}, & y \text { is bounded on } J\end{cases}
$$

where ${ }^{c} D_{0^{+}}^{\alpha}$ is the Caputo fractional derivative of order $\alpha, f: J \times \mathbb{R} \rightarrow \mathbb{R}$ is a is a continuous function and $y_{0} \in \mathbb{R}$. The main approach is based on Schauder's fixed point theorem. my results is to generalize the previous work.

This article concerns the existence of solutions of boundary value problem for fractional differential equations on unbounded interval. We consider the boundary value problem

$$
I_{0^{+}}^{2-\alpha} y\left(0^{+}\right)=y_{0}
$$

$D_{0^{+}}^{\alpha}$ denote Riemann-Liouville fractional derivative introduced, $1<\alpha \leq 2$. The operator $I_{0^{+}}^{2-\alpha}$ denotes the left-sided Riemann-Liouville fractional integral, the state $y(\cdot)$ takes value in a Banach space $E, f$ : $(0, \infty) \times E \rightarrow E$ will be specified in later sections and $\left(y_{0}, y_{\infty}\right) \in E \times E$.

This paper is organized in the following way. In Section 2, we give some general results and preliminaries and in Section 3, we present existence results for the problem (1.1)-(1.3), using the Mönch's fixed point theorem combined with the technique of measure of noncompactness. Finally an illustrative example will be presented in Section 4.

\section{Preliminary Results}

In this section, we introduce some notation and technical results which are used throughout this paper. Let $I \subset J$ be a compact interval and denote by $C(I, E)$ the Banach space of continuous functions $y: I \rightarrow E$ with the usual norm

$$
\|y\|_{\infty}=\sup \{\|y(t)\|, t \in I\}
$$

$L^{1}(J, E)$ the space of $E$-valued Bochner integrable functions on $J$ with the norm

$$
\|f\|_{L^{1}}=\int_{0}^{+\infty}\|f(t)\| d t
$$


We consider the space of functions

$$
C_{\alpha}([0, \infty), E)=\left\{y \in C(J, E): \lim _{t \rightarrow 0^{+}} t^{2-\alpha} y(t) \text { exists and finite }\right\} .
$$

For $y \in C_{\alpha}((0, \infty), E)$, we define $y_{\alpha}$ by

$$
y_{\alpha}(t)= \begin{cases}t^{2-\alpha} y(t), & t \in(0, \infty), \\ \lim _{t \longrightarrow 0} t^{2-\alpha} y(t), & t=0 .\end{cases}
$$

It is clear that $y_{\alpha} \in C([0, \infty), E)$.

We consider the following Banach space

$$
X_{\alpha}([0, \infty), E)=\left\{y \in C_{\alpha}([0, \infty), E): \lim _{t \rightarrow \infty} \frac{t^{2-\alpha} y(t)}{1+t^{\alpha}} \text { exists and finite }\right\}
$$

A norm in this space is given by

$$
\|y\|_{\alpha}=\sup _{t \in J} \frac{t^{2-\alpha}\|y(t)\|}{1+t^{\alpha}} .
$$

We begin with some definitions from the theory of fractional calculus. Let $\alpha>0, n=\lceil\alpha\rceil+1$ (the least integer greater than or equal to $\alpha)$ and $h \in C(J, E)$.

Definition 2.1. [18].

(1) The Riemann-Liouville fractional integral of the function $h$ of order $\alpha$ is defined by

$$
I_{0+}^{\alpha} h(t)=g_{\alpha}(t) * h(t)=\int_{0}^{t} g_{\alpha}(t-s) h(s) d s, t>0,
$$

where $*$ denotes convolution and $g_{\alpha}(t)=t^{\alpha-1} / \Gamma(\alpha)$.

(2) The Riemann-Liouville fractional derivative of the function $h$ of order $\alpha$ is defined by

$$
D_{0+}^{\alpha} h(t)=\frac{d^{n}}{d t^{n}}\left(g_{n-\alpha}(t) * h(t)\right)
$$

for all $t>0$. Where $\Gamma$ is the gamma function.

For the existence of solutions for the problem (1.1)-(1.3), we need the following auxiliary lemmas.

Lemma 2.1. [26] Let $\alpha>0$, then the differential equation

$$
D_{0^{+}}^{\alpha} h(t)=0
$$

has solutions $h(t)=c_{1} t^{\alpha-1}+c_{2} t^{\alpha-2}+\ldots+c_{n} t^{\alpha-n}$, for some $c_{i} \in \mathbb{R}, i=1 \ldots n$, where $n=[\alpha]+1$.

Lemma 2.2. [26] Let $\alpha>0$, then

$$
I_{0^{+}}^{\alpha} D_{0^{+}}^{\alpha} h(t)=h(t)+c_{1} t^{\alpha-1}+c_{2} t^{\alpha-2}+\ldots+c_{n} t^{\alpha-n}
$$

for some $c_{i} \in \mathbb{R}, \quad i=0, \ldots, n$, where $n=[\alpha]+1$. 
Remark 2.1. For $\alpha>0, k>-1$, we have

$$
I_{0+}^{\alpha} t^{k}=\frac{\Gamma(k+1)}{\Gamma(\alpha+k+1)} t^{\alpha+k} \text { and } D_{0+}^{\alpha} t^{k}=\frac{\Gamma(k+1)}{\Gamma(k-\alpha+1)} t^{k-\alpha}, t>0,
$$

giving in particular $D_{0+}^{\alpha} t^{\alpha-m}=0, m=1, \ldots, n$, where $n$ is the smallest integer greater than or equal to $\alpha$.

Remark 2.2. If $h$ is suitabe function (see for instance [18, 20,23]), we have the composition relations $D_{0+}^{\alpha} I_{0+}^{\alpha} h(t)=h(t), \alpha>0$ and $D_{0+}^{\alpha} I_{0+}^{k} h(t)=I_{0+}^{k-\alpha} h(t), k>\alpha>0, t>0$.

We note that $\gamma, \gamma_{C}$ and $\gamma_{X_{\alpha}}$ the Kuratowski noncompactness measure of bounded sets in the spaces $E, C(I, E)$ and $X_{\alpha}$, respectively. As for the definition of the Kuratowski noncompactness measure, we refer to references $[5,17]$. The following properties of the Kuratowski measure of noncompactness and Mönch fixed point theorem are needed for our discussion.

Lemma 2.3. If $H \subset C(I, E)$ is bounded and equicontinuous, then $\gamma(H(t))$ is continuous on $I$ and

$$
\gamma_{C}(H)=\max _{t \in I} \gamma(H(t)), \gamma\left(\left\{\int_{I} x(t) d t: x \in H\right\}\right) \leq \int_{I} \gamma(H(t)) d t
$$

where $H(t)=\{x(t): x \in H\}$.

Theorem 2.1. [2,24] Let $D$ be a bounded, closed and convex subset of a Banach space $E$ such that $0 \in D$ , and let $N$ be a continuous mapping of $D$ into itself. If the implication

$$
V=\overline{\operatorname{conv}} N(V) \text { or } V=N(V) \cup\{0\} \Longrightarrow \gamma(V)=0,
$$

holds for every subset $V$ of $D$, then $N$ has a fixed point.

\section{MAIN RESUlT}

We will need to introduce the following hypotheses which are assumed here after.

$\left(H_{1}\right)$ There exists a nonnegative functions $a, b \in C\left(J, \mathbb{R}^{+}\right)$such that

$$
\|f(t, u)\| \leq a(t)+t^{2-\alpha} b(t)\|u\| \text { for all } t \in J \text { and } u \in E,
$$

where

$$
\int_{0}^{\infty}\left(1+t^{\alpha}\right) b(t) d t<\Gamma(\alpha), \int_{0}^{\infty} a(t) d t<\infty .
$$

$\left(H_{2}\right) \forall t \in(0, L], \forall x, y \in E:\|f(t, x)-f(t, y)\| \leq \frac{t^{2-\alpha}}{1+t^{\alpha}}\|x-y\|$.

$\left(H_{3}\right)$ There exists nonnegative function $\ell \in L^{1}\left(J, \mathbb{R}^{+}\right)$such that for each nonempty, bounded set $\Omega \subset$ $X_{\alpha}(J, E)$

$$
\gamma(f(t, \Omega(t))) \leq t^{2-\alpha} \ell(t) \gamma(\Omega(t)), \quad \text { for all } t \in J
$$

where

$$
\int_{0}^{\infty}\left(1+t^{\alpha}\right) \ell(t) d t \leq \Gamma(\alpha)
$$


Definition 3.1. A function $y \in X_{\alpha}([0,+\infty))$ is said to be solution of the problem (1.1)-(1.3) if $y$ satisfies the equation $D_{0^{+}}^{\alpha} y(t)=f(t, y(t))$ and the conditions $(1.2-1.3)$.

Lemma 3.1. Let $1<\alpha<2$ and let $h: J \rightarrow E$ be continuous. A function $y$ is a solution of the fractional integral equation

$$
y(t)=\frac{1}{\Gamma(\alpha)}\left[y_{\infty}-\int_{0}^{\infty} h(t) d t\right] t^{\alpha-1}+\frac{y_{0}}{\Gamma(\alpha-1)} t^{\alpha-2}+\frac{1}{\Gamma(\alpha)} \int_{0}^{t}(t-s)^{\alpha-1} h(s) d s
$$

if and only if $y$ is solution of the problem

$$
\begin{gathered}
D_{0^{+}}^{\alpha} y(t)=h(t), \quad t \in J=(0,+\infty), \\
I_{0^{+}}^{2-\alpha} y\left(0^{+}\right)=y_{0}, \\
D_{0^{+}}^{\alpha-1} y(\infty)=y_{\infty} .
\end{gathered}
$$

Proof. Assume that $y$ satisfies the problem (3.2)-(3.4). We may apply Lemma 2.2 to reduce equation (3.2) to an equivalent integral equation

$$
y(t)=c_{1} t^{\alpha-1}+c_{2} t^{\alpha-2}+I_{0^{+}}^{\alpha} h(t)
$$

for some $c_{1}, c_{2} \in \mathbb{R}$. Applying $I_{0^{+}}^{2-\alpha}$ to both side of (3.5), we have

$$
I_{0^{+}}^{2-\alpha} y(t)=c_{1} I_{0^{+}}^{2-\alpha} t^{\alpha-1}+c_{2} I_{0^{+}}^{2-\alpha} t^{\alpha-2}+I_{0^{+}}^{2-\alpha} I_{0^{+}}^{\alpha} h(t) .
$$

From Remark 2.1, we then get

$$
I_{0^{+}}^{2-\alpha} y(t)=\frac{c_{1} \Gamma(\alpha)}{\Gamma(2)} t+c_{2} \Gamma(\alpha-1)+\frac{1}{\Gamma(2)} \int_{0}^{t}(t-s) h(s) d s .
$$

As $t \longrightarrow 0$, we obtain

$$
c_{2}=\frac{y_{0}}{\Gamma(\alpha-1)} .
$$

Applying $D_{0^{+}}^{\alpha-1}$ to both side of (3.5), we have

$$
D_{0^{+}}^{\alpha-1} y(t)=c_{1} D_{0^{+}}^{\alpha-1} t^{\alpha-1}+c_{2} D_{0^{+}}^{\alpha-1} t^{\alpha-2}+D_{0^{+}}^{\alpha-1} I_{0^{+}}^{\alpha} h(t) .
$$

From Remark 2.1 and Remark 2.2, we then get

$$
D_{0^{+}}^{\alpha-1} y(t)=c_{1} \Gamma(\alpha)+\frac{1}{\Gamma(1)} \int_{0}^{t} h(s) d s .
$$


Hence

$$
c_{1}=\frac{1}{\Gamma(\alpha)}\left(y_{\infty}-\int_{0}^{\infty} h(t) d t\right)
$$

Thus, we have

$$
y(t)=\frac{1}{\Gamma(\alpha)}\left[y_{\infty}-\int_{0}^{\infty} h(t) d t\right] t^{\alpha-1}+\frac{y_{0}}{\Gamma(\alpha-1)} t^{\alpha-2}+\frac{1}{\Gamma(\alpha)} \int_{0}^{t}(t-s)^{\alpha-1} h(s) d s .
$$

Conversely. The proof is simple.

Consider the operator $N: X_{\alpha}([0, \infty), E) \rightarrow X_{\alpha}([0, \infty), E)$ defined by

$$
\begin{aligned}
N(y)(t) & =\frac{y_{\infty}}{\Gamma(\alpha)} t^{\alpha-1}+\frac{y_{0}}{\Gamma(\alpha-1)} t^{\alpha-2}-\frac{1}{\Gamma(\alpha)} \int_{0}^{t}\left[t^{\alpha-1}-(t-s)^{\alpha-1}\right] f(s, y(s)) d s \\
& -\frac{1}{\Gamma(\alpha)} \int_{t}^{\infty} t^{\alpha-1} f(s, y(s)) d s .
\end{aligned}
$$

The following several lemmas present some properties of the operator $N$, which are necessary for the proof of our main result.

Lemma 3.2. Suppose that conditions $\left(H_{1}\right)$ and $\left(H_{2}\right)$ are valid. Then the operator $N$ is bounded and continuous.

Proof. For $y \in X_{\alpha}([0, \infty), E)$, it is easy to deduce from $\left(H_{1}\right)$, and that $N y \in X_{\alpha}(J, E)$. Furthermore, $\left(H_{1}\right)$ guarantees that

$$
\begin{aligned}
& \frac{t^{2-\alpha}\|N(y)(t)\|}{1+t^{\alpha}} \leq \frac{\left\|y_{\infty}\right\|}{\Gamma(\alpha)}+\frac{\left\|y_{0}\right\|}{\Gamma(\alpha-1)}+\frac{1}{\Gamma(\alpha)} \int_{0}^{\infty}\|f(s, y(s))\| d s \\
& \leq \frac{\left\|y_{\infty}\right\|}{\Gamma(\alpha)}+\frac{\left\|y_{0}\right\|}{\Gamma(\alpha-1)}+\frac{\|y\|_{\alpha}}{\Gamma(\alpha)} \int_{0}^{\infty}\left(1+t^{\alpha}\right) b(t) d t+\frac{1}{\Gamma(\alpha)} \int_{0}^{\infty} a(t) d t .
\end{aligned}
$$

Hence, $N: X_{\alpha}(J, E) \rightarrow X_{\alpha}(J, E)$ is bounded. Next we prove that $N$ is continuous. Let $\left\{y_{n}\right\}_{n=1}^{\infty} \subset X_{\alpha}(J, E)$ and $y \in X_{\alpha}(J, E)$ such that $y_{n} \rightarrow y$ as $n \rightarrow \infty$. Then, $\left\{y_{n}\right\}_{n=1}^{\infty}$ is a bounded set of $X_{\alpha}(J, E)$, i.e. there exists $M>0$ such that $\left\|y_{n}\right\|_{\alpha} \leq M$ for $n>1$. We also have by taking limit that $\|y\|_{\alpha} \leq M$. In view of condition $\left(H_{1}\right)$, for any $\varepsilon>0$, there exists $L>0$ such that

$$
\int_{L}^{\infty} a(t) d t<\frac{\Gamma(\alpha)}{6} \varepsilon, \int_{L}^{\infty}\left(1+t^{\alpha}\right) b(t) d t<\frac{\Gamma(\alpha) \varepsilon}{6 M} \varepsilon
$$

and there exists $\widetilde{N} \in \mathbb{N}$ such that, for all $n \geq \tilde{N}$, we have

$$
\left\|f\left(s, y_{n}(s)\right)-(s, y(s))\right\|<\frac{\Gamma(\alpha)}{3 L} \varepsilon .
$$

Therefore, for all $t \in J$ and $n>\widetilde{N}$, we can obtain from

$$
\begin{aligned}
\frac{t^{2-\alpha}}{1+t^{\alpha}}\left\|N\left(y_{n}\right)(t)-N(y)(t)\right\| & \leq \frac{1}{\Gamma(\alpha)} \int_{0}^{t}\left\|f\left(s, y_{n}(s)\right)-f(s, y(s))\right\| d s \\
& +\frac{1}{\Gamma(\alpha)} \int_{t}^{\infty}\left\|f\left(s, y_{n}(s)\right)-f(s, y(s))\right\| d s .
\end{aligned}
$$


If $t \leq L$ and $n>\widetilde{N}$, we can obtain from

$$
\begin{aligned}
& \frac{t^{2-\alpha}}{1+t^{\alpha}}\left\|N\left(y_{n}\right)(t)-N(y)(t)\right\| \leq \frac{1}{\Gamma(\alpha)} \int_{0}^{t}\left\|f\left(s, y_{n}(s)\right)-f(s, y(s))\right\| d s \\
& +\frac{1}{\Gamma(\alpha)} \int_{t}^{\infty}\left\|f\left(s, y_{n}(s)\right)-f(s, y(s))\right\| d s \\
& \leq \frac{1}{\Gamma(\alpha)} \int_{0}^{t}\left\|f\left(s, y_{n}(s)\right)-f(s, y(s))\right\| d s \\
& +\frac{1}{\Gamma(\alpha)}\left[\int_{t}^{L}\left\|f\left(s, y_{n}(s)\right)-f(s, y(s))\right\| d s+\int_{L}^{\infty}\left\|f\left(s, y_{n}(s)\right)-f(s, y(s))\right\| d s\right] \\
& \leq \frac{2}{\Gamma(\alpha)} \int_{0}^{L}\left\|f\left(s, y_{n}(s)\right)-f(s, y(s))\right\| d s+\frac{2 M}{\Gamma(\alpha)} \int_{L}^{\infty}\left(1+s^{\alpha}\right) b(s) d s+\frac{2}{\Gamma(\alpha)} \int_{L}^{\infty} a(s) d s \\
& \leq \frac{\varepsilon}{3}+\frac{\varepsilon}{3}+\frac{\varepsilon}{3}=\varepsilon .
\end{aligned}
$$

The case when $t>L$ and $n>\tilde{N}$ is treated similarly. Thus we conclude that

$$
\left\|y_{n}-y\right\|_{\alpha} \rightarrow 0 \text { as } n \rightarrow \infty
$$

namely, $N$ is continuous and the conclusion of the lemma follows.

Lemma 3.3. Let condition $\left(H_{1}\right)$ be satisfied and $B$ be a bounded subset of $X_{\alpha}(J, E)$. Then

(i) $\frac{t^{2-\alpha} N(B)(t)}{1+t^{\alpha}}$ is equicontinuous on any compact interval of $J$.

(ii) For given $\varepsilon>0$, there exists a constant $N_{1}>0$ such that $\left\|\frac{t_{1}^{2-\alpha} N(y)\left(t_{1}\right)}{1+t_{1}^{\alpha}}-\frac{t_{2}^{2-\alpha} N(y)\left(t_{2}\right)}{1+t_{2}^{\alpha}}\right\|<\varepsilon$ for any $t_{1}, t_{2} \geq N_{1}$ and $y(.) \in B$.

Proof. We have

$$
N y(t)=\frac{y_{\infty}-\int_{0}^{\infty} f(t, y(t)) d t}{\Gamma(\alpha)} t^{\alpha-1}+\frac{y_{0}}{\Gamma(\alpha-1)} t^{\alpha-2}+\frac{1}{\Gamma(\alpha)} \int_{0}^{t}(t-s)^{\alpha-1} f(s, y(s)) d s
$$

In view of condition $\left(H_{1}\right)$ and the boundedness of $B$, there exists $M>0$ such that

$$
\int_{0}^{\infty}\|f(t, y(t))\| d t \leq M \text { for any } y \in B
$$


In order to prove $(i)$, let the constant $r$ be such that $\|y\|_{\alpha} \leq r$ for any $y \in B$, and without loss of generality, let $[a, b] \subset J$ be a compact interval and $t_{1}, t_{2} \in[a, b]$ with $t_{1}<t_{2}$. Then

$$
\begin{aligned}
& \left\|\frac{t_{1}^{2-\alpha} N(y)\left(t_{1}\right)}{1+t_{1}^{\alpha}}-\frac{t_{2}^{2-\alpha} N(y)\left(t_{2}\right)}{1+t_{2}^{\alpha}}\right\| \leq \frac{\left\|y_{\infty}\right\|+M}{\Gamma(\alpha)}\left|\frac{t_{1}}{1+t_{1}^{\alpha}}-\frac{t_{2}}{1+t_{2}^{\alpha}}\right|+\frac{\left\|y_{0}\right\|}{\Gamma(\alpha-1)}\left|\frac{1}{1+t_{1}^{\alpha}}-\frac{1}{1+t_{2}^{\alpha}}\right| \\
& +\frac{1}{\Gamma(\alpha)}\left\|\int_{0}^{t_{1}}\left(t_{1}-s\right)^{\alpha-1} f(s, y(s)) d s-\int_{0}^{t_{2}}\left(t_{2}-s\right)^{\alpha-1} f(s, y(s)) d s\right\| \\
& \leq \frac{\left\|y_{\infty}\right\|+M}{\Gamma(\alpha)}\left|\frac{t_{1}}{1+t_{1}^{\alpha}}-\frac{t_{2}}{1+t_{2}^{\alpha}}\right|+\frac{\left\|y_{0}\right\|}{\Gamma(\alpha-1)}\left|\frac{1}{1+t_{1}^{\alpha}}-\frac{1}{1+t_{2}^{\alpha}}\right| \\
& +\frac{1}{\Gamma(\alpha)} \int_{0}^{t_{1}}\left|\left(t_{2}-s\right)^{\alpha-1}-\left(t_{1}-s\right)^{\alpha-1}\right|\|f(s, y(s))\| d s \\
& +\frac{1}{\Gamma(\alpha)} \int_{t_{1}}^{t_{2}}\left(t_{2}-s\right)^{\alpha-1}\|f(s, y(s))\| d s \\
& \leq \frac{\left\|y_{\infty}\right\|+M}{\Gamma(\alpha)}\left|\frac{t_{1}}{1+t_{1}^{\alpha}}-\frac{t_{2}}{1+t_{2}^{\alpha}}\right|+\frac{\left\|y_{0}\right\|}{\Gamma(\alpha-1)}\left|\frac{1}{1+t_{1}^{\alpha}}-\frac{1}{1+t_{2}^{\alpha}}\right| \\
& +\frac{1}{\Gamma(\alpha)} \int_{0}^{t_{1}}\left|\left(t_{2}-s\right)^{\alpha-1}-\left(t_{1}-s\right)^{\alpha-1}\right| a(s) d s \\
& +\frac{r}{\Gamma(\alpha)} \int_{0}^{t_{1}}\left|\left(t_{2}-s\right)^{\alpha-1}-\left(t_{1}-s\right)^{\alpha-1}\right|\left(1+s^{\alpha}\right) b(s) d s+\frac{1}{\Gamma(\alpha)} \int_{t_{1}}^{t_{2}}\left(t_{2}-s\right)^{\alpha-1} a(s) d s \\
& +\frac{r}{\Gamma(\alpha)} \int_{t_{1}}^{t_{2}}\left(t_{2}-s\right)^{\alpha-1}\left(1+s^{\alpha}\right) b(s) d s \\
& \leq \frac{\left\|y_{\infty}\right\|+M}{\Gamma(\alpha)}\left|\frac{t_{1}}{1+t_{1}^{\alpha}}-\frac{t_{2}}{1+t_{2}^{\alpha}}\right|+\frac{\left\|y_{0}\right\|}{\Gamma(\alpha-1)}\left|\frac{1}{1+t_{1}^{\alpha}}-\frac{1}{1+t_{2}^{\alpha}}\right| \\
& +\frac{a^{*}+b^{*} r}{\Gamma(\alpha)}\left(\int_{0}^{t_{1}}\left(t_{2}-s\right)^{\alpha-1}-\left(t_{1}-s\right)^{\alpha-1} d s\right)+\frac{a^{*}+b^{*} r}{\Gamma(\alpha)} \int_{t_{1}}^{t_{2}}\left(t_{2}-s\right)^{\alpha-1} d s \\
& +\frac{2 b^{*} r}{\Gamma(\alpha)}\left(\int_{0}^{t_{2}}\left(t_{2}-s\right)^{\alpha-1} s^{\alpha} d s-\int_{0}^{t_{1}}\left(t_{1}-s\right)^{\alpha-1} s^{\alpha} d s\right) \\
& \leq \frac{\left\|y_{\infty}\right\|+M}{\Gamma(\alpha)}\left|\frac{t_{1}}{1+t_{1}^{\alpha}}-\frac{t_{2}}{1+t_{2}^{\alpha}}\right|+\frac{\left\|y_{0}\right\|}{\Gamma(\alpha-1)}\left|\frac{1}{1+t_{1}^{\alpha}}-\frac{1}{1+t_{2}^{\alpha}}\right| \\
& +\frac{a^{*}+b^{*} r}{\Gamma(1+\alpha)}\left(t_{2}^{\alpha}-t_{1}^{\alpha}-\left(t_{2}-t_{1}\right)^{\alpha}\right)+\frac{a^{*}+b^{*} r}{\Gamma(1+\alpha)}\left(t_{2}-t_{1}\right)^{\alpha}+\frac{2 b^{*} r \mathcal{B}(\alpha, \alpha+1)}{\Gamma(\alpha)}\left(t_{2}^{2 \alpha}-t_{1}^{2 \alpha}\right),
\end{aligned}
$$

where $a^{*}=\max _{t \in[a, b]} a(t)$ and $b^{*}=\max _{t \in[a, b]} b(t)$. As $t_{2} \rightarrow t_{1}$ the right-hand side of the above inequality tends to zero. Then $\frac{t^{2-\alpha} N(B)(t)}{1+t^{\alpha}}$ is equicontinuous on $[a, b]$.

Next we verify assertion $(i i)$. Let $\varepsilon>0$, we heve

$$
\begin{aligned}
& \left\|\frac{t_{1}^{2-\alpha} N(y)\left(t_{1}\right)}{1+t_{1}^{\alpha}}-\frac{t_{2}^{2-\alpha} N(y)\left(t_{2}\right)}{1+t_{2}^{\alpha}}\right\| \leq \frac{\left\|y_{\infty}\right\|+M}{\Gamma(\alpha)}\left|\frac{t_{1}}{1+t_{1}^{\alpha}}-\frac{t_{2}}{1+t_{2}^{\alpha}}\right|+\frac{\left\|y_{0}\right\|}{\Gamma(\alpha-1)}\left|\frac{1}{1+t_{1}^{\alpha}}-\frac{1}{1+t_{2}^{\alpha}}\right| \\
& +\frac{1}{\Gamma(\alpha)}\left\|\int_{0}^{t_{1}} \frac{t_{1}^{2-\alpha}\left(t_{1}-s\right)^{\alpha-1}}{1+t_{1}^{\alpha}} f(s, y(s)) d s-\int_{0}^{t_{2}} \frac{t_{2}^{2-\alpha}\left(t_{2}-s\right)^{\alpha-1}}{1+t_{2}^{\alpha}} f(s, y(s)) d s\right\| .
\end{aligned}
$$


It is sufficient to prove that

$$
\left\|\int_{0}^{t_{1}} \frac{t_{1}^{2-\alpha}\left(t_{1}-s\right)^{\alpha-1}}{1+t_{1}^{\alpha}} f(s, y(s)) d s-\int_{0}^{t_{2}} \frac{t_{2}^{2-\alpha}\left(t_{2}-s\right)^{\alpha-1}}{1+t_{2}^{\alpha}} f(s, y(s)) d s\right\| \leq \varepsilon .
$$

Relation (3.6) yields that there exits $N_{0}>0$ such that

$$
\int_{N_{0}}^{\infty}\|f(t, y(t))\| d t \leq \frac{\varepsilon}{3} \text { for any } y \in B
$$

On the other hand, since $\lim _{t \rightarrow \infty} \frac{t^{2-\alpha}\left(t-N_{0}\right)^{\alpha-1}}{1+t^{\alpha}}=0$, there exists $N_{1}>N_{0}$ such that, for any $t_{1}, t_{2} \geq N_{1}$ and $s \in\left[0, N_{0}\right]$, we have

$$
\left|\frac{t_{2}^{2-\alpha}\left(t_{2}-s\right)^{\alpha-1}}{1+t_{2}^{\alpha}}-\frac{t_{1}^{2-\alpha}\left(t_{1}-s\right)^{\alpha-1}}{1+t_{1}^{\alpha}}\right|<\frac{\varepsilon}{3 M} .
$$

Now taking $t_{1}, t_{2} \geq N_{1}$, from $(3.7)$, (3.8) we can arrive at

$$
\begin{aligned}
& \left\|\int_{0}^{t_{1}} \frac{t_{1}^{2-\alpha}\left(t_{1}-s\right)^{\alpha-1}}{1+t_{1}^{\alpha}} f(s, y(s)) d s-\int_{0}^{t_{2}} \frac{t_{2}^{2-\alpha}\left(t_{2}-s\right)^{\alpha-1}}{1+t_{2}^{\alpha}} f(s, y(s)) d s\right\| \\
& \leq \int_{0}^{N_{1}}\left|\frac{t_{2}^{2-\alpha}\left(t_{2}-s\right)^{\alpha-1}}{1+t_{2}^{\alpha}}-\frac{t_{1}^{2-\alpha}\left(t_{1}-s\right)^{\alpha-1}}{1+t_{1}^{\alpha}}\right|\|f(s, y(s))\| d s \\
& +\int_{N_{1}}^{t_{1}} \frac{t_{1}^{2-\alpha}\left(t_{1}-s\right)^{\alpha-1}}{1+t_{1}^{\alpha}}\|f(s, y(s))\| d s+\int_{N_{1}}^{t_{2}} \frac{t_{2}^{2-\alpha}\left(t_{2}-s\right)^{\alpha-1}}{1+t_{2}^{\alpha}}\|f(s, y(s))\| d s \\
& <\frac{\varepsilon}{3 M} \int_{0}^{\infty}\|f(s, y(s))\| d s+2 \int_{N_{1}}^{\infty}\|f(s, y(s))\| d s<\varepsilon .
\end{aligned}
$$

Therefore, we complete the proof of lemma 3.3.

Lemma 3.4. [25] Suppose that condition $\left(H_{1}\right)$ holds and $B$ is a bounded subset of $X_{\alpha}(J, E)$. Then $\gamma_{X_{\alpha}}(N(B))=\sup _{t \in J} \gamma\left(\frac{t^{2-\alpha} N(B)(t)}{1+t^{\alpha}}\right)$.

Now we are in a position to give the main result of this work. Let

$$
B=\left\{y \in X_{\alpha}([0, \infty), E):\|y\|_{\alpha} \leq R\right\}
$$

Theorem 3.1. Suppose that conditions $\left(H_{1}\right),\left(H_{2}\right)$ and $\left(H_{3}\right)$ are valid. If

$\left(H_{4}\right)$

$$
R>\frac{\left\|y_{\infty}\right\|+(\alpha-1)\left\|y_{0}\right\|+\int_{0}^{\infty} a(t) d t}{\Gamma(\alpha)-\int_{0}^{\infty}\left(1+t^{\alpha}\right) b(t) d t}
$$

Then the problem (1.1)-(1.3) has at least one solution.

Proof. First we transform problem (1.1)-(1.3) into a fixed point problem. Consider the operator $N$ : $X_{\alpha}([0, \infty), E) \rightarrow X_{\alpha}([0, \infty), E)$ defined by

$$
\begin{aligned}
N(y)(t) & =\frac{y_{\infty}}{\Gamma(\alpha)} t^{\alpha-1}+\frac{y_{0}}{\Gamma(\alpha-1)} t^{\alpha-2}-\frac{1}{\Gamma(\alpha)} \int_{0}^{t}\left[t^{\alpha-1}-(t-s)^{\alpha-1}\right] f(s, y(s)) d s \\
& -\frac{1}{\Gamma(\alpha)} \int_{t}^{\infty} t^{\alpha-1} f(s, y(s)) d s
\end{aligned}
$$


From Lemma 3.1, the fixed points of $N$ are solutions to (1.1)-(1.3). We shall show that $N$ satisfies the assumptions of Mönch fixed point theorem (Theorem 2.1).

Then we can derive that $N: B \rightarrow B$. Indeed, for any $y \in B$, by condition $\left(H_{1}\right)$ we get

$$
\begin{aligned}
& \left\|\frac{t^{2-\alpha} N(y)(t)}{1+t^{\alpha}}\right\| \leq \frac{\left\|y_{\infty}\right\|}{\Gamma(\alpha)}+\frac{\left\|y_{0}\right\|}{\Gamma(\alpha-1)}+\frac{1}{\Gamma(\alpha)} \int_{0}^{\infty}\|f(t, y(t))\| d t \\
& \left.\leq \frac{(1}{\Gamma(\alpha)}\left(\left\|y_{\infty}\right\|+(\alpha-1)\left\|y_{0}\right\|+\int_{0}^{\infty} a(t) d t+R \int_{0}^{\infty}\left(1+t^{\alpha}\right)\right) b(t) d t\right)<R .
\end{aligned}
$$

Hence, from $\left(H_{4}\right)$ we have $\|N y\|_{\alpha} \leq R$, and we conclude that $N: B \rightarrow B$. Clearly $B$ is a bounded, convex and closed subset of $X_{\alpha}([0, \infty), E)$, together with Lemma 3.2 we know that $N: B \rightarrow B$ is continuous. Finally we need to prove the following implication

$$
V \subset \overline{\operatorname{conv}}\{N(V) \cup\{0\}\} \Longrightarrow \gamma_{X_{\alpha}}(V)=0 \text {, for any } V \subset B \text {. }
$$

Let $V \subset B$ such that $V \subset \overline{\operatorname{conv}}\{N(V) \cup\{0\}\}$ and $t \in J$, we choose $\xi>0$ and $n>0$ such that $\xi<t<n$. For each $y \in V$, we consider

$$
\begin{aligned}
& N_{\xi, n}(y)(t)=\frac{y_{\infty}}{\Gamma(\alpha)} t^{\alpha-1}+\frac{y_{0}}{\Gamma(\alpha-1)} t^{\alpha-2}+\frac{1}{\Gamma(\alpha)} \int_{\xi}^{t}\left[t^{\alpha-1}-(t-s)^{\alpha-1}\right] f(s, y(s)) d s \\
& +\frac{1}{\Gamma(\alpha)} \int_{t}^{n}(t-s)^{\alpha-1} f(s, y(s)) d s .
\end{aligned}
$$

Then from $\left(H_{1}\right)$, we obtain that

$$
\begin{aligned}
& \frac{t^{2-\alpha}}{1+t^{\alpha}}\left\|N_{\xi, n}(y)(t)-N(y)(t)\right\| \leq \frac{1}{\Gamma(\alpha)} \int_{0}^{\xi}\|f(t, y(t))\| d t+\frac{1}{\Gamma(\alpha)} \int_{n}^{\infty}\|f(t, y(t))\| d t \\
& \left.\left.\leq \frac{1}{\Gamma(\alpha)}\left(\int_{0}^{\xi} a(t) d t+R \int_{0}^{\xi}\left(1+t^{\alpha}\right)\right) b(t) d t+\int_{n}^{\infty} a(t) d t+R \int_{n}^{\infty}\left(1+t^{\alpha}\right)\right) b(t) d t\right),
\end{aligned}
$$

this shows that $H_{d}\left(\frac{t^{2-\alpha} N_{\xi, n}(V)(t)}{1+t^{\alpha}}, \frac{t^{2-\alpha} N(V)(t)}{1+t^{\alpha}}\right) \rightarrow 0$ as $\xi \rightarrow 0$ and $n \rightarrow \infty, t \in J$. Where $H_{d}$ denotes the Hausdorff metric in space $E$. By the prorerty of noncompactness mearure we get

$$
\lim _{\xi \rightarrow 0, n \rightarrow \infty} \gamma\left(\frac{t^{2-\alpha} N_{\xi, n}(V)(t)}{1+t^{\alpha}}\right)=\gamma\left(\frac{t^{2-\alpha} N(V)(t)}{1+t^{\alpha}}\right)
$$

From lemma 3.3, the set $\left\{\frac{t^{2-\alpha} N(V)(t)}{1+t^{\alpha}}\right\} \subset X_{\alpha}([0, \infty), E)$ is equicontinuous on any compact of $J$. By $\left(H_{1}\right)$, il is easy to know that $\{f(., y()):. y \in V\}$ is equicontinuous on $[\xi, n]$. Moreover $\{f(., y()):. y \in V\}$ is bounded 
on $[\xi, n]$, by $\left(H_{1}\right)$. Using Lemma 2.3 , Lemma 3.4 and $\left(H_{3}\right)$, we arrive at

$$
\begin{aligned}
\gamma\left(\frac{t^{2-\alpha} N_{\xi, n} V(t)}{1+t^{\alpha}}\right) & \leq \frac{1}{\Gamma(\alpha)} \int_{\xi}^{n}\left(1+t^{\alpha}\right) \ell(t) \gamma\left(\frac{t^{2-\alpha} V(t)}{1+t^{\alpha}}\right) d t \\
& \leq \frac{1}{\Gamma(\alpha)} \int_{\xi}^{n}\left(1+t^{\alpha}\right) \ell(t) \gamma\left(\frac{t^{2-\alpha} N(V)(t)}{1+t^{\alpha}}\right) d t \\
& \leq \frac{1}{\Gamma(\alpha)} \int_{\xi}^{n}\left(1+t^{\alpha}\right) \ell(t) \gamma_{X_{\alpha}}(N(V)) d t
\end{aligned}
$$

From (3.9), we know that

$$
\gamma\left(\frac{t^{2-\alpha} N(V)(t)}{1+t^{\alpha}}\right) \leq \frac{1}{\Gamma(\alpha)} \int_{0}^{\infty}\left(1+t^{\alpha}\right) \ell(t) \gamma_{X_{\alpha}}(N(V)) d t
$$

Thus

$$
\gamma_{X_{\alpha}}(N(V)) \leq \frac{1}{\Gamma(\alpha)} \int_{0}^{\infty}\left(1+t^{\alpha}\right) \ell(t) \gamma_{X_{\alpha}}(N(V)) d t
$$

Consequently, by condition $\left(H_{3}\right)$. We get $\gamma_{X_{\alpha}}(N(V))=0$; that is $\gamma_{X_{\alpha}}(V)=0$. From the theorem 2.1 , we conclude that $N$ has a fixed point $y \in B$ which is a solution of problem (1.1)-(1.3).

\section{EXAMPLE}

We consider the following problem.

$$
\begin{gathered}
D^{\frac{3}{2}} y(t)=\left(\frac{\sqrt{t} y_{n}(t)}{\left(1+t^{\frac{3}{2}}\right) e^{10 t}}+\frac{2 t)}{\left(1+t^{2}\right)^{2}}\right)_{n=1}^{\infty}, \quad t \in J=(0,+\infty) \\
I_{0^{+}}^{\frac{1}{2}} y(t)=y_{0} \\
D_{0^{+}}^{\frac{1}{2}} y(\infty)=y_{\infty} .
\end{gathered}
$$

Let

$$
E=\left\{\left(y_{1}, \ldots, y_{n}, \ldots\right): \sup \left|y_{n}\right|<\infty\right\}
$$

with the norm $\|y\|=\sup _{n}\left|y_{n}\right|$, then $E$ is a Banach space and problem (4.1)-(4.3) can be regarded as a problem of the form (1.1)-(1.3), with

$$
\alpha=\frac{3}{2} \text { and } f(t, y(t))=\left(f\left(t, y_{1}(t)\right), \ldots, f\left(t, y_{n}(t)\right), \ldots\right),
$$

where

$$
f\left(t, y_{n}(t)\right)=\frac{\sqrt{t} y_{n}(t)}{\left(1+t^{\frac{3}{2}}\right) e^{10 t}}+\frac{2 t}{\left(1+t^{2}\right)^{2}}, n \in \mathbb{N}^{*}
$$


We shall verify the conditions $\left(H_{1}\right)-\left(H_{3}\right)$. Evidently, $f$ is continuous in $J \times E$ and

$$
\|f(t, y(t))\| \leq \frac{\sqrt{t}}{\left(1+t^{\frac{3}{2}}\right) e^{10 t}}\|y(t)\|+\frac{2 t}{\left(1+t^{2}\right)^{2}} .
$$

With the aid of simple computation we find that

$$
\int_{0}^{\infty} e^{-5 t} d t=\frac{1}{10}<\Gamma\left(\frac{3}{2}\right) \text { and } \int_{0}^{\infty} \frac{2 t}{\left(1+t^{2}\right)^{2}} d t=1<\infty
$$

Finally, we verify condition $\left(H_{3}\right)$. For any bounded set $B \subset E$, we have

$$
f(t, B(t))=\frac{\sqrt{t}}{\left(1+t^{\frac{3}{2}}\right) e^{5 t}} B(t)+\left\{\frac{2 t}{\left(1+t^{2}\right)^{2}}\right\} .
$$

Then

$$
\gamma\left(f(t, B(t)) \leq \frac{\sqrt{t}}{\left(1+t^{\frac{3}{2}}\right) e^{5 t}} \gamma(B(t)) .\right.
$$

Since $\int_{0}^{\infty} e^{-10 t} d t=0.1<\Gamma\left(\frac{3}{2}\right)$, we conclude that condition $\left(H_{3}\right)$ is satisfied. Therefore, Theorem 3.1 ensures that problem (4.1)-(4.3) has a solution.

Acknowledgments: The authors would like to express their thanks to the editor and anonymous referees for his/her comments that improved the quality of the paper.

Conflicts of Interest: The author(s) declare that there are no conflicts of interest regarding the publication of this paper.

\section{ReferenCes}

[1] R. P. Agarwal, B. Hedia and M. Beddani, Structure of solution sets for implulsive fractional differential equations,Fract. Calc. Appl. Anal. 9 (2018), 15-36.

[2] R. P. Agarwal, M. Meehan and D. O'Regan, Fixed Point Theory and Applications , Cambridge Tracts in Mathematics, 141 , Cambridge University Press, Cambridge, 2001.

[3] B. Ahmad, Existence of solutions for irregular boundary value problems of nonlinear fractional differential equations, Appl. Math. Lett. 23 (2010), 390-394.

[4] A. Arara, M. Benchohra, N. Hamidi and J.J. Nieto, Fractional order differential equations on an unbounded domain, Nonlinear Anal., Theory Methods Appl. 72 (2010), 580-586.

[5] Z.B. Bai and H.S. Lü, Positive solutions for boundary value problem of nonlinear fractional differential equation, J. Math. Anal. Appl. 311 (2005), 495-505.

[6] K. Balachandran and J.Y. Park, Nonlocal Cauchy problem for abstract fractional semilinear evolution equations, Nonlinear Anal., Theory Methods Appl. 71 (2009), 4471-4475.

[7] K. Balachandran and J.J. Trujillo, The nonlocal Cauchy problem for nonlinear fractional integrodifferential equations in Banach spaces, Nonlinear Anal., Theory Methods Appl. 72 (2010), 4587-4593.

[8] K. Balachandran, S. Kiruthika and J.J. Trujillo, Existence results for fractional impulsive integrodifferential equations in Banach spaces, Commun. Nonlinear Sci. Numer. Simul. 16 (2011), 1970-1977.

[9] M. Benchohra, S. Hamani and S.K. Ntouyas, Boundary value problems for differential equations with fractional order and nonlocal conditions, Nonlinear Anal., Theory Methods Appl. 71 (2009), 2391-2396. 
[10] L. Byszewski, Existence and uniqueness of mild and classical solutions of semilinear functional-differential evolution nonlocal Cauchy problem, Selected problems of mathematics, 50th Anniv. Cracow Univ. Technol. Anniv. Issue 6, Cracow Univ. Technol. Krakow, (1995), 25-33.

[11] L. Byszewski and V.Lakshmikantham, Theorem about the existence and uniqueness of a solution of a nonlocal abstract Cauchy problem in a Banach space, Appl. Anal. 40 (1991), 11-19.

[12] G. Christopher, Existence and uniqueness of solutions to a fractional difference equation with nonlocal conditions, Computers Math. Appl. 61(2011), 191-202.

[13] W. Cheung, J. Ren, P.J.Y. Wong and D. Zhao, Multiple positive solutions for discrete nonlocal boundary value problems, J. Math. Anal. Appl. 330 (2007), 900-915.

[14] K. Deng, Exponential decay of solutions of semilinear parabolic equations with nonlocal initial conditions, J. Math. Anal. Appl. 179 (1993), 630-637.

[15] A. M. A. El-Sayed, Nonlinear functional differential equations of arbitrary orders, Nonlinear Anal., Theory Methods Appl. 33 (1998), 181-186.

[16] A. M. A. El-Sayed and A. G. Ibrahim, Multivalued fractional differential equations, Appl. Math. Comput. 68 (1995), 15-25.

[17] D.J. Guo, V. Lakshmikantham and X. Liu, Nonlinear Integral Equations in Abstract Spaces, Kluwer Academic Publishers, Dordrecht, 1996.

[18] A.A. Kilbas, H.M. Srivastava and J.J. Trujillo, Theory and Applications of Fractional Differential Equations, Elsevier B.V. Amsterdam, 2006.

[19] G. M. N'Guérékata, A Cauchy problem for some fractional abstract differential equation with non local conditions, Nonlinear Anal., Theory Methods Appl. 70 (2009), 1873-1876.

[20] I. Podlubny, Fractional Differential Equations, in: Mathematics in Science and Engineering, vol. 198, Academic Press, New York, London, Toronto, 1999.

[21] H.A.H. Salem, On the fractional calculus in abstract spaces and their applications to the Dirichlet-type problem of fractional order, Comput. Math. Appl. 59 (2010), 1278-1293.

[22] H.A.H. Salem, Multi-term fractional differential equation in reflexive Banach space, Math. Comput. Model. 49 (2009), 829-834.

[23] S.G. Samko, A.A. Kilbas and O.I. Marichev, Fractional Integrals and Derivatives: Theory and Applications, Gordon and Breach, Yverdon, 1993.

[24] S. Szufla, On the application of measure of noncompactness to existence theorems, Rend. Sem. Mat. Univ. Padova. 75 (1986), 1-14.

[25] X. Su, Solutions to boundary value problem of fractional order on unbounded domains in a Banach space, Nonlinear Anal., Theory Methods Appl. 74 (2011), 2844-2852.

[26] S. Zhang, Positive solutions for boundary-value problems of nonlinear fractional differential equations, Electron. J. Differ. Equations, 2006 (2006), 36. 